\title{
The European Spallation Source: Designing a Sustainable Research Infrastructure for Europe
}

\author{
John Womersley
}

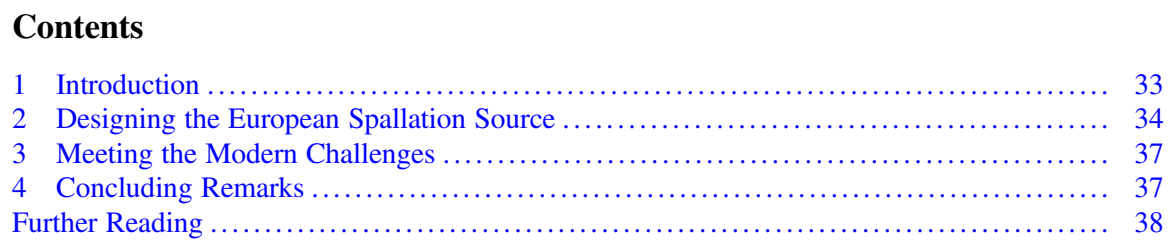

\section{Introduction}

The paper briefly outlines some of the key challenges in building sustainable support for any science megaproject, using the European Spallation Source (ESS) as an example (Fig. 1). Beyond the project's imminent socio-economic impact the essay also reflects on the broader question of how public investments in large-scale "Big Science" projects can tackle the present global inequalities by reshaping the current forces of globalization, offering more opportunities for participation and empowering marginalized groups that often feel excluded.

Any science megaproject needs to have the following in place to succeed:

- a solid science case

- technical R\&D carried out to reduce key risks, and cost estimates that are understood

- a solid project management plan

- a credible funding and governance plan

- good stakeholder engagement and support, and

- a compelling investment case

Let's look at each of these questions in turn.

\footnotetext{
J. Womersley $(\square)$

The European Spallation Source, Lund, Sweden

e-mail: John.Womersley@ess.eu 


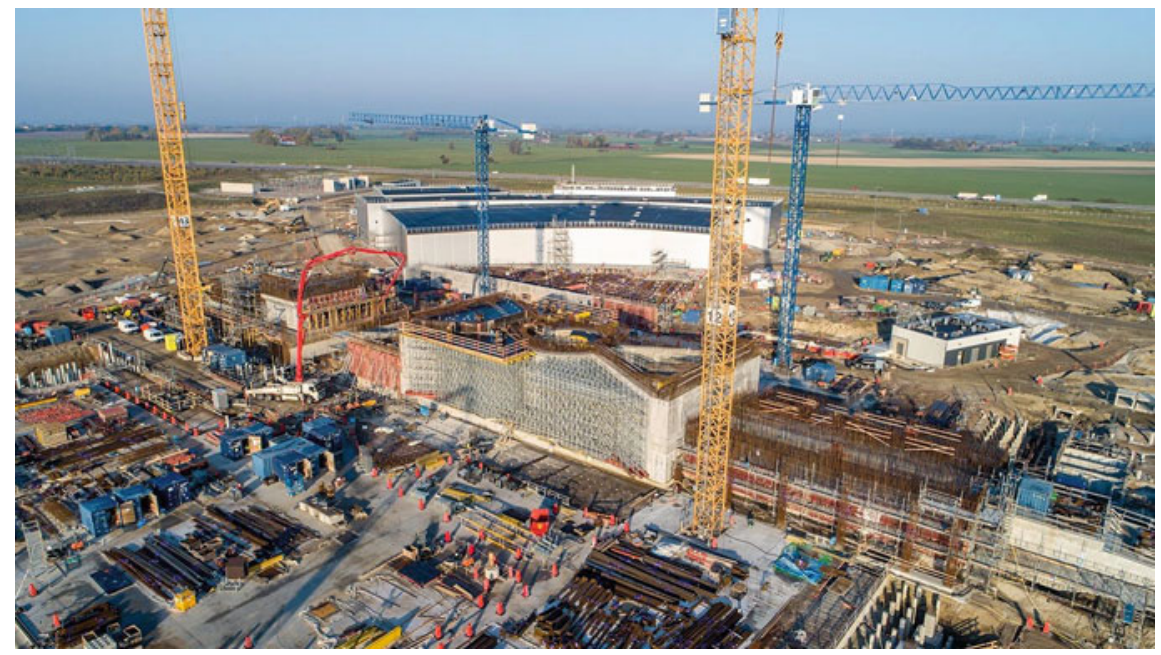

Fig. 1 ESS under construction in Lund, Sweden. The central concrete structure will house the neutron production target

\section{Designing the European Spallation Source}

For ESS, the investment case in a nutshell is that scientific and technological innovation is essential to address both the global challenges of energy, climate, environment, healthcare, and the economic and societal challenges of stalled productivity and long term wage stagnation. The particular contribution that ESS will make is to use intense beams of neutrons to allow scientists to study the structure of materials and molecules: where are the atoms and what do they do? This knowledge underpins the development of new materials, new drugs, new processes, and new energy technologies.

The vision of ESS then is to build and operate the world's most powerful neutron source, enabling scientific breakthroughs in research related to materials, energy, health and the environment, and addressing some of the most important societal challenges of our time.

There is a long and successful legacy of neutron scattering worldwide, based primarily on the use of research reactors as neutron sources. However, there is an effective maximum flux of neutrons that can be obtained from a reactor if the chain reaction is to be controllable. The way to generate much higher fluxes, to study smaller samples or make measurements faster, is to use an accelerator-driven source that produces neutrons through spallation reactions from a high- $Z$ target (tungsten in the case of ESS) when struck with a. proton beam of energy $1-2 \mathrm{GeV} / \mathrm{c}^{2}$. The spallation neutrons emerge with high energies characteristic of nuclear processes and therefore need to be slowed down until their quantum mechanical wavelengths are of the order of $10^{-10} \mathrm{~m}$, the spacing between atoms in the kind of samples that we wish to study. This is done by elastically scattering the neutrons; ESS uses a polarised 
liquid hydrogen moderator to achieve this. The neutrons are then guided to experimental stations where the samples to be studied are located, and the scattered neutrons are detected and measured by large instrumentation arrays. From the angles, energies and phases of the outgoing neutrons, the structure and composition of the sample can be inferred.

Some areas where technical advances and R\&D were needed to deliver the ESS performance goals were:

- the superconducting accelerator and in particular the use of spoke cavities in the low-energy section

- the neutron production target which is based on a helium cooled rotating wheel

- the neutron moderator design which is two to three times more efficient in terms of useful neutrons per unit beam power and

- the development of solid state boron-based neutron detectors to replace increasingly scarce helium-3.

All of these innovations required early-stage R\&D funding to allow their development to the point where the risk of proceeding with the full project was acceptable.

A few numbers set the scale of the project. The construction cost (in 2013 prices) is $1843 \mathrm{M} €$. At $5 \mathrm{MW}$ ( $2 \mathrm{MW}$ at start of operation) ESS will operate the world's most powerful particle accelerator-a $400 \mathrm{~m}$ long superconducting linac- to drive the spallation target. Neutrons will be detected in 15 experimental stations, each optimised for different types of measurements. The combination of increased accelerator power, more efficient neutron generation and better resolution instrumentation will yield an increase in sensitivity of a factor of 20 on average compared with today's best facilities of this type. We anticipate roughly 800 experiments will be carried out per year, by several thousand visiting scientists.

Groundbreaking took place in Lund, in southern Sweden, in 2014, and the project is now $60 \%$ complete. 2019 is the peak year of construction activity. Major installation activities are underway and we have entered the initial operations phase, with the first components of the particle accelerator in commissioning. First science with external users will take place in 2023.

To establish a new facility like this on a green-field site requires not just constructing the scientific infrastructure but also the organisation to build and operate it. ESS has recruited over 450 staff from 54 nationalities to work on the project, roughly $40 \%$ from Sweden, $40 \%$ from the rest of Europe and 20\% from the rest of the world, including from as far away as Japan, Australia, the USA and Canada.

The Legal structure of ESS is a European Research Infrastructure Consortium (ERIC) which was conceived as a lighter weight alternative to setting up a treaty organisation for European inter-governmental scientific facilities. The ESS ERIC was established in 2015. There are 13 member states. The two host Countries, Sweden and Denmark, have agreed to pay a $47.5 \%$ share of the construction costs, mainly in cash; the non-hosts pay the remainder but roughly $70 \%$ in kind rather than in cash. 


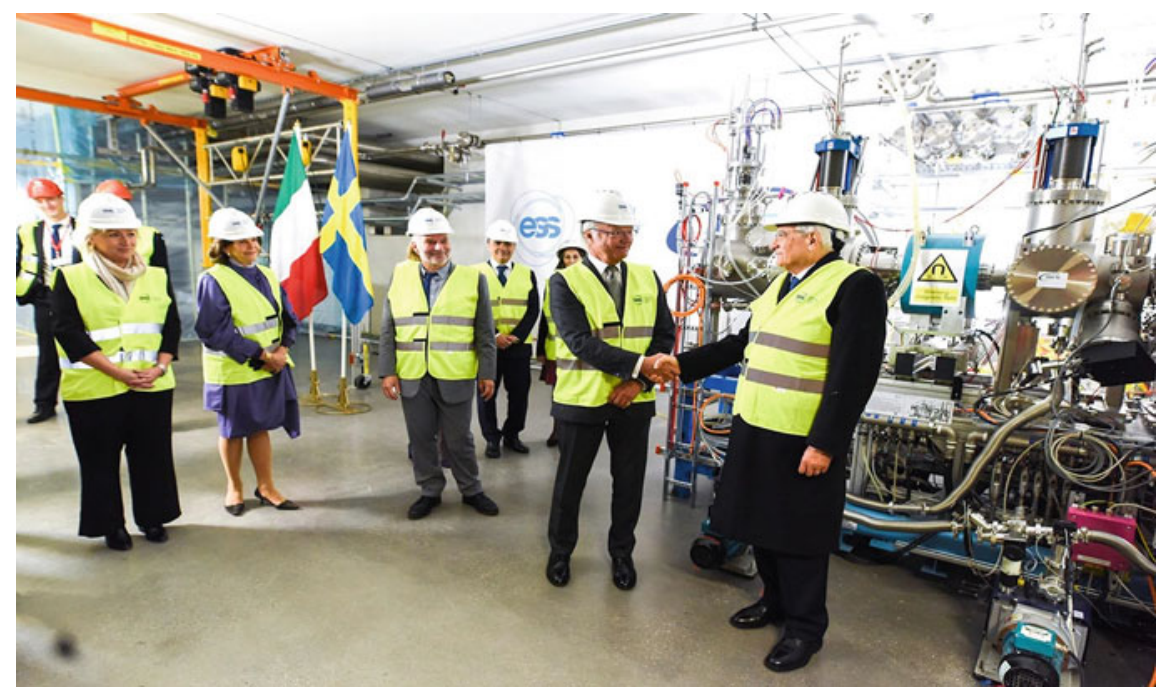

Fig. 2 One of the first major in-kind deliveries at ESS. The proton source, built at INFN in Catania in Italy, is officially inaugurated in Lund by HE the President of Italy and HM the King of Sweden

By in-kind contributions we mean significant technical workpackages procured or constructed in partner countries rather than centrally. The partner holds and manages the risk. This can be a project management challenge, but nowadays is close to a political necessity. It helps avoid the situation where the host region benefits greatly and the others just pay cash. At ESS, 70\% in kind for the non-hosts, translates to about $35 \%$ overall, which is just about manageable as there are sufficient cash contributions to cover the needs of project contingency. In contrast, the ITER Project, where the in-kind fraction approaches $90 \%$, has proved very hard to manage (Fig. 2).

ESS has established a network of in-kind partners in each of its 13 members. Doing so has benefited the project by providing access to well-established technical expertise and engineering resources in national laboratories such as Saclay, RAL, Jülich and avoided the need to establish any manufacturing capability for accelerator and target components in Lund. Managing the in-kind supply chain, though, including procurements, quality control, schedule and eventual systems integration at ESS, does require significant effort.

Any large capital investment needs to show that it is following good project management practice. In ESS's case we have chosen to mirror the requirements of the US Department of Energy's Office of Project Management Oversight and Assessment (DOE order 413.3B) which is usually seen as best practice in the big science domain. Any project must have a resource-loaded schedule, sufficient contingency, a clear change control process, and good financial controls. ESS uses Primavera as our project planning tool and the ESS schedule contains over 20,000 linked activities. The critical path is clearly understood and proactively managed. 


\section{Meeting the Modern Challenges}

Up to now we've discussed requirements that would have applied to any major science investment over the last few decades. But a new facility being built in 2019 must also reflect today's expectations. It must meet modern environmental expectations: ESS is a green construction site with no waste to landfill and construction machinery powered by biofuels. We will purchase all our electrical power from renewable sources, and waste heat from our electrical machinery will be recovered into the Lund district heating system. Modern research facilities must also meet modern data handling expectations.

ESS will provide support for the full computing, software and analysis chain at its data centre in Copenhagen and will operate an open data model as part of the European Open Science Cloud. Modern facilities are also expected to help the translation of research from academia to industry; to this end a science and innovation campus is being developed in the land area between ESS and the neighbouring MAX IV light source.

We have now briefly reviewed most of the items listed in the checklist described at the start-having a good science case, technical R\&D and cost estimates understood, project management, a credible funding and governance plan, and a compelling investment case.

\section{Concluding Remarks}

Finally, a few closing observations. Firstly, why do big projects fail to get started? In 2016 the European Strategy Forum for Research Infrastructures (which I chaired at that time) reviewed the implementation progress of the projects on its roadmap. We found that inadequate stakeholder engagement and lack of a credible funding plan were the biggest barriers to implementation-much more so than any weakness of the science case.

Stakeholders include (but are not limited to) the General Public, media and "opinion formers," students, educators (the STEM skills pipeline), university bosses, other areas of science, Members of Parliament, Science Ministers, Finance Ministers, Opposition political parties, Local and regional politicians, Civil Servants, economists. All of these are multiplied by the number of member countries, each with their own science strengths, industrial profile, media and decision-making culture.

Especially where the government stakeholders are concerned, there has been a shift in emphasis since the end of the Cold War from investing in science to promote normative values (implicitly in contrast to the values of the competing bloc) such as openness, the cultural value of science, science for peace and democracy, and to promote international collaboration, towards market or social values such as science as a driver of prosperity, jobs, innovation, startups and as a solution to grand 
challenges for climate and the environment. Scientists were probably rather happier with the cold war values, as they made fewer demands on them-but investment decisions are now made firmly on the market and social impact of projects.

The biggest economic challenges of our time include globalisation, which together with automation and new technologies is leading to fewer good jobs in developed countries, to low growth, stagnant wages, angry people on the streets and populist politics. No one really has the answer to this, but there is a general consensus that scientific innovation and STEM skills are key-or at least economies and people that have these skills will be better positioned to deal with these challenges. So what is our project going to do to help? This is a question that all of us as scientists, but also policy-makers, citizens and especially students who will be the future users of these facilities need to be able to confidently answer.

\section{Further Reading}

1. European Spallation Source - 2018 Activity Report: https://europeanspallationsource.se/sites/ default/files/files/document/2019-08/ESS\%20Activity\%20Report\%202018.pdf

2. European Strategy Forum on Research Infrastructures - Roadmap 2016 https://www.esfri.eu/ sites/default/files/20160308_ROADMAP_single_page_LIGHT.pdf

3. Neutron users in Europe: Facility-based insights and scientific trends: https:// europeanspallationsource.se/publications

\section{Full Presentation}

https://indico.cern.ch/event/727555/contributions/3476197/attachments/1868049/3072618/ JohnWomersley.pdf

Open Access This chapter is licensed under the terms of the Creative Commons Attribution 4.0 International License (http://creativecommons.org/licenses/by/4.0/), which permits use, sharing, adaptation, distribution and reproduction in any medium or format, as long as you give appropriate credit to the original author(s) and the source, provide a link to the Creative Commons licence and indicate if changes were made.

The images or other third party material in this chapter are included in the chapter's Creative Commons licence, unless indicated otherwise in a credit line to the material. If material is not included in the chapter's Creative Commons licence and your intended use is not permitted by statutory regulation or exceeds the permitted use, you will need to obtain permission directly from the copyright holder.

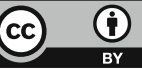

